\title{
Uniqueness and non-uniqueness of static black holes in higher dimensions
}

\author{
${ }^{(a)}$ Gary W. Gibbons, ${ }^{(b)}$ Daisuke Ida and ${ }^{(b)}$ Tetsuya Shiromizu \\ ${ }^{(a)}$ DAMTP, Centre for Mathematical Sciences, The University of Cambridge, \\ Wilberforce Road, Cambridge CB3 OWA, United Kingdom \\ ${ }^{(b)}$ Research Center for the Early Universe (RESCEU), The University of Tokyo, Tokyo 113-0033, Japan
}

\begin{abstract}
We prove a uniqueness theorem for asymptotically flat static charged dilaton black hole solutions in higher dimensional space-times. We also construct infinitely many non-asymptotically flat regular static black holes on the same space-time manifold with the same spherical topology. An application to the uniqueness of a certain class of flat $p$-branes is also given.
\end{abstract}

With the development of string theory, black holes in higher dimensional space-times have come to play a fundamental role in physics [1]. Furthermore, the possibility of black hole production in high energy experiments has recently been suggested in the context of the so-called $\mathrm{TeV}$ gravity [2]. A $\mathrm{TeV}$-size black hole in $\mathrm{TeV}$ gravity is small enough to be well approximated by an asymptotically flat black hole in higher dimensions. To predict phenomenological results, we need reliable knowledge about higher dimensional black holes. However, some essential features of black hole theory have not so far been fully explored in many recent discussions. Among these, the equilibrium problem for black holes is one of most important issues. The final equilibrium state of the black hole is known to drastically simplify in the case of four spacetime dimensions because of the uniqueness properties of static or stationary black hole solutions. The uniqueness theorem for the vacuum black hole has been well established in four-dimensional space-times [3 5] (See also Ref. [6] for comprehensive review). Although this no hair property is fundamental to the nature of black holes, it is at the same time a quite non-trivial result derived from the Einstein equations. Remarkably, five-dimensional stationary vacuum black holes are not unique; there is a Myers-Perry solution [1], which is a generalization of the Kerr solution to arbitrary dimensions, while Emparan and Reall [7] have recently found five-dimensional rotating black ring solutions with the same angular momenta and mass but now the event horizon homeomorphic to $S^{2} \times S^{1}$. In the static case, such a counter-example has not yet been presented. The only known asymptotically flat static vacuum black hole is the $n$-dimensional hyperspherically symmetric Schwarzschild-Tangherlini solution [8]. In this paper we shall show that there are no others. However it is interesting to note that, as we shall expand upon below, if one drops the condition of asymptotic flatness but still insists that the space-time has the same topology as that of the Schwarschild-Tanghelerlini solution, then the uniqueness property fails badly. There exist discrete infinities of solutions.

In this paper we shall show that static charged dilatonic non-extreme black holes with a certain dilaton coupling constant are also unique. (See also [15] for an earlier paper on the vacuum case in the context of Riemannian geometry).
Our work was motivated by models in string theory where gauge fields often play an essential role. Another important motivation is to embark on the programme of proving the uniqueness of static black $p$-brane solutions. These are $(n+p)$-dimensional spacetimes invariant under the action of a $p$-dimensional Abelian translation group. Reduction to $n$ spacetime dimensions produces a black hole solution of gravity coupled to one or more scalars and an electric 2 -form or dual magnetic $(n-2)$-form field strength. We shall return to this aspect of our work in a later publication.

The main tools we use are method developed by Masood-ul-Alam [16] to deal with charged dilaton black holes in $3+1$ spacetime dimensions and a new method of our own (introduced independently by 15]) based on the use of totally umbillic hypersurfaces. The latter is an essential ingredient becaues existing $3+1$ methods do apply. It is also essential to assume strict asymptotic flatness because using some Einstein metrics on $S^{n-2}, n=7, \ldots, 11$ due to Bohm we can construct infinitely many non-asymptotically flat solutions on manifolds wuth the same topology. In addition, just as in $3+1$ dimensions, we also have to assume that the surface gravity is non-zero (non-extreme), otherwise one has multi-black holes solutions.

The first half of our work parallels that of Bunting and Masood-ul-Alam 湫, so that we shall only briefly describe it here.

We start with the Lagrangian in $n$-dimensional spacetime

$$
L={ }^{n} R-2(\partial \phi)^{2}-e^{-\alpha \phi} F^{2},
$$

where $F$ is the Maxwell field and the dilaton coupling constant is set to $\alpha=[8(n-3) /(n-2)]^{1 / 2}$. In general, the metric of an $n$-dimensional static space-time has the form

$$
d s^{2}=-V^{2} d t^{2}+g_{i j} d x^{i} d x^{j}
$$

\footnotetext{
*Note that our proof presented here is only true for this choice for $\alpha$. If not, for example, Eqs (17), (18) and (20) are not correct and then proof is not correct.
} 
where $V$ and $g_{i j}$ are independent of $t$ and they are regarded as quantities on the $t=$ const. hypersurface $\Sigma$. The event horizon $H$ is a Killing horizon located at the level set $V=0$, which is assumed to be non-degenerate. In fact non-degeneracy follows from Smarr's formula relating the mass, surface gravity and area of the horizon. Then the static field equations become

$$
\begin{aligned}
\nabla^{2} V & =\frac{\alpha^{2} e^{-\alpha \phi}}{16 V}(\nabla \psi)^{2} \\
\nabla^{2} \phi & =\frac{\alpha e^{-\alpha \phi}}{8 V^{2}}(\nabla \psi)^{2}-\frac{\nabla V \cdot \nabla \phi}{V} \\
\nabla^{2} \psi & =\nabla \psi \cdot\left(\alpha \nabla \phi+\frac{\nabla V}{V}\right)
\end{aligned}
$$

and

$$
\begin{aligned}
R_{i j}= & \frac{\nabla_{i} \nabla_{j} V}{V}+2 \nabla_{i} \phi \nabla_{j} \phi \\
& -\frac{e^{-\alpha \phi}}{2 V^{2}}\left[\nabla_{i} \psi \nabla_{j} \psi-\frac{(\nabla \psi)^{2}}{n-2} g_{i j}\right],
\end{aligned}
$$

where $\nabla$ and $R_{i j}$ denote covariant derivative and the Ricci tensor defined on $\left(\Sigma, g_{i j}\right)$, respectively, and $\psi$ is the electrostatic potential such that $F=d \psi \wedge d t$.

In asymptotically flat space-times, one can find an appropriate coordinate system in which the metric, dilaton and electrostatic potential have asymptotic expansions of the form

$$
\begin{aligned}
V & =1-\frac{\mu}{r^{n-3}}+O\left(1 / r^{n-2}\right), \\
g_{i j} & =\left(1+\frac{2}{n-3} \frac{\mu}{r^{n-3}}\right) \delta_{i j}+O\left(1 / r^{n-2}\right), \\
e^{\alpha \phi / 2} & =1-\frac{Q^{2}}{\mu r^{n-3}}+O\left(1 / r^{n-2}\right), \\
\psi & =\frac{2^{5 / 2} Q}{\alpha r^{n-3}}+O\left(1 / r^{n-2}\right),
\end{aligned}
$$

respectively, where $\mu, Q=$ const. represent the ADM mass and the electric charge (up to constant factors), respectively, and $r:=\sqrt{\sum_{i}\left(x^{i}\right)^{2}}$. We assume the nonextremal condition $\mu>|Q|$.

Consider the following two conformal transformations

$$
\hat{g}_{i j}^{ \pm}=\left(e^{-\alpha \phi / 2} \lambda_{1}^{ \pm} \lambda_{2}^{ \pm}\right)^{4 /(n-3)} g_{i j}
$$

where

$$
\begin{aligned}
& \lambda_{1}^{ \pm}:=\frac{1 \pm V e^{\alpha \phi / 2}}{2}+2^{-5 / 2} \alpha \psi, \\
& \lambda_{2}^{ \pm}:=\frac{1 \pm V e^{\alpha \phi / 2}}{2}-2^{-5 / 2} \alpha \psi
\end{aligned}
$$

Then we have two manifolds $\left(\Sigma^{ \pm}, g_{i j}^{ \pm}\right)$. On $\Sigma^{+}$, the asymptotic behavior of the metric becomes

$$
\hat{g}_{i j}^{+}=\delta_{i j}+O\left(1 / r^{n-2}\right) .
$$

On $\Sigma^{-}$, we have

$$
\begin{aligned}
\hat{g}_{i j}^{-} d x^{i} d x^{j}= & \left(\frac{\mu^{2}-Q^{2}}{2 \mu}\right)^{4 /(n-3)}\left(d \varrho^{2}+\varrho^{2} d \Omega_{n-2}^{2}\right) \\
& +O\left(\varrho^{5}\right) .
\end{aligned}
$$

where $d \Omega_{n-2}^{2}$ denotes the round sphere metric and $\varrho:=$ $1 / r$ has been defined. Pasting $\left(\Sigma^{ \pm}, g_{i j}^{ \pm}\right)$across the level set $V=0$ and adding a point $\{p\}$ at $\varrho=0$, we can construct a complete regular surface $\hat{\Sigma}=\Sigma^{+} \cup \Sigma^{-} \cup\{p\}$. The Ricci scalar (sometimes called the scalar curvature) $\hat{R}$ on $\Sigma^{ \pm}$becomes

$$
\hat{R}=\frac{4}{\alpha^{2}}\left|\hat{\nabla} \ln \frac{\lambda_{1}^{ \pm}}{\lambda_{2}^{ \pm}} \mp \frac{\alpha e^{-\alpha \phi / 2}}{2^{3 / 2} V} \hat{\nabla} \psi\right|^{2}+\frac{16}{\alpha^{2}} \frac{\hat{\nabla}^{2} \tau_{ \pm}}{\tau_{ \pm}},
$$

where $\tau_{ \pm}:=e^{\alpha \phi / 2}\left(\lambda_{1}^{ \pm} \lambda_{2}^{ \pm}\right)^{-1 / 2}$ has been defined. Note that the last term in (17) has no definite sign. Nevertheless we can still generalize the standard positive energy theorem due to Witten to cover this new situation.

Following [16] we consider the Witten spinor $\Psi$ (an asymptotically constant spinor satisfying $\gamma^{i} D_{i} \Psi=0$ ) on $\left(\hat{\Sigma}^{ \pm}, g_{i j}^{ \pm}\right)$. Then we have a divergence identity

$$
\begin{aligned}
& \hat{\nabla} \cdot\left[\hat{\nabla}|\Psi|^{2}-\frac{8}{\alpha^{2}}|\Psi|^{2} \hat{\nabla} \ln \frac{\tau_{ \pm}}{\sigma_{ \pm}}\right] \\
= & {\left[\frac{2}{\alpha^{2}}\left|\hat{\nabla} \ln \frac{\lambda_{1}^{ \pm}}{\lambda_{2}^{ \pm}} \mp \frac{\alpha e^{-\alpha \phi / 2}}{2^{3 / 2} V} \hat{\nabla} \psi\right|^{2}\right.} \\
& \left.+\frac{(n-2)(n-4)}{2(n-3)^{2}}\left|\hat{\nabla} \ln \frac{\tau_{ \pm}}{\sigma_{ \pm}}\right|^{2}\right]|\Psi|^{2} \\
& +2\left|D \Psi-\frac{4}{\alpha^{2}}\left(\hat{\nabla} \ln \frac{\tau_{ \pm}}{\sigma_{ \pm}}\right) \Psi\right|^{2},
\end{aligned}
$$

where $\sigma_{ \pm}:=\left(1 \pm V e^{-\alpha \phi / 2}\right)^{-1}$ has been defined. We have used

$$
\begin{aligned}
\hat{\nabla}^{2}|\Psi|^{2} & =\frac{\hat{R}}{2}|\Psi|^{2}+2|D \Psi|^{2}, \\
\frac{\hat{\nabla}^{2} \sigma_{ \pm}}{\sigma_{ \pm}} & =2 \frac{\hat{\nabla} \sigma_{ \pm}}{\sigma_{ \pm}} \cdot\left(\frac{\hat{\nabla} \sigma_{ \pm}}{\sigma_{ \pm}}-\frac{\hat{\nabla} \tau_{ \pm}}{\tau_{ \pm}}\right)
\end{aligned}
$$

Integrating Eq. (18) over $\hat{\Sigma}$, positive mass theorem can be proven. In other words, the l.h.s. represents the total gravitational mass on $\hat{\Sigma}$ and it is positive definite from the expression in the r.h.s.. However, Eq. (15) implies that the total gravitational mass is zero on $\hat{\Sigma}$. This means that the each terms in the r.h.s of Eq. (18) vanish:

$$
\begin{aligned}
\hat{\nabla}_{i} \ln \frac{\lambda_{1}^{ \pm}}{\lambda_{2}^{ \pm}} & = \pm \frac{\alpha e^{-\alpha \phi / 2}}{2^{3 / 2} V} \hat{\nabla}_{i} \psi, \\
\hat{\nabla}_{i} \ln \frac{\tau_{ \pm}}{\sigma_{ \pm}} & =0 \\
D_{i} \Psi & =0 .
\end{aligned}
$$


Equations (21), (22) imply that the level sets of $V, \phi$ and $\psi$ conincide. Furthermore, the spinor equation (23) implies that $\hat{\Sigma}$ is the flat space: $\hat{g}_{i j}=\delta_{i j}$ Then, Eq. (17) implies that $\tau_{ \pm}$is a harmonic function on the flat space: $\nabla_{0}^{2} \tau_{ \pm}=0$, where $\nabla_{0}$ denotes the flat connection.

We shall now demonstrate that the conformally transformed event horizon $\hat{H}$ is a geometric sphere in $\hat{\Sigma}$. We choose $V$ as a local coordinate in a neighbour hood $U \subset \Sigma$. Let $\left\{x^{A}\right\}$ be coordinates on level sets of $V$ such that their trajectries are orthogonal to each level set. Then, the metric on $\Sigma$ can be written in the form

$$
g=\rho^{2} d V^{2}+h_{A B} d x^{A} d x^{B},
$$

where $\rho^{2}:=(\nabla V)^{2}$. Since $\Sigma$ is conformally flat, the Riemann invariant has a simple expression in this coordinate system:

$$
\begin{aligned}
{ }^{n} R_{I J K L}{ }^{n} R^{I J K L}= & R_{i j k l} R^{i j k l}+4 R_{0 i 0 j} R^{0 i 0 j} \\
= & \frac{4(n-2)}{(n-3) V^{2} \rho^{2}}\left[k_{A B} k^{A B}+k^{2}\right. \\
& \left.+2 \mathcal{D}_{A} \rho \mathcal{D}^{A} \rho\right]
\end{aligned}
$$

where $\mathcal{D}_{A}$ denotes the covariant derivative on each level set of $V$, and $k_{A B}$ is the second fundamental form of the level set.

The requirement that the event horizon $H$ is a regular surface leads to the condition

$$
\begin{aligned}
& \left.k_{A B}\right|_{H}=0, \\
& \left.\mathcal{D}_{A} \rho\right|_{H}=0 .
\end{aligned}
$$

In particular, $H$ is a totally geodesic surface in $\Sigma$.

Let us consider how the event horizon is embedded into the base space $\left(\hat{\Sigma}, \delta_{i j}\right)$. In terms of the smooth function $\tau:=\tau_{ \pm}$, we can adopt the following local expression for the flat space

$$
\delta_{i j} d x^{i} d x^{j}=\hat{\rho}^{2} d \tau^{2}+\hat{h}_{A B} d x^{A} d x^{B} .
$$

The event horizon is located at some $\tau=\tau_{H}=$ const. surface $\hat{H}$. Then, the extrinsic curvature $\hat{k}_{A B}$ of the level set $\tau=$ const. can be expressed as

$$
\begin{aligned}
\hat{k}_{A B}= & e^{\alpha \phi /(n-3)} \tau^{-4 /(n-3)} k_{A B} \\
& +\frac{2}{n-3} \frac{\left(e^{-\alpha \phi / 4} \tau\right)^{2(n-1) /(n-3)}}{\rho} \hat{h}_{A B} .
\end{aligned}
$$

Thus we obtain

$$
\hat{k}_{A B}=\left.\frac{2}{n-3} \frac{\left(e^{-\alpha \phi / 4} \tau\right)^{2(n-1) /(n-3)}}{\rho}\right|_{H} \hat{h}_{A B} .
$$

on $\hat{H}$. In other words, the embedding of $\hat{H}$ into the Euclidean $(n-1)$-space is totally umbilical. It is known that such a embedding must be hyperspherical [9], namely each connected component of $\hat{H}$ is a geometric sphere with a certain radius. The embedding of a hypersphere into the Euclidean space is known to be rigid [10], which means that we can always locate one connected component of $\tilde{H}$ at the $r=r_{0}$ surface of $\tilde{\Sigma}$ without loss of generality. If there is only a single horizon, we have a boundary value problem for the Laplace equation $\nabla_{0}^{2} \tau=0$ on the base space $\Omega:=E^{n-1} \backslash B^{n-1}$ with the Dirichlet boundary conditions. Such a solution must be spherically symmetric, so that it is given by the solutions found in [17].

One may remove the assumption of the single horizon as follows. Consider the evolution of the level surface in Euclidean space. From the Gauss equation in Euclidean space one obtains the evolution equation for the shear $\hat{\sigma}_{A B}:=\hat{k}_{A B}-\hat{k} \hat{h}_{A B} /(n-2)$ :

$$
\begin{aligned}
£_{\hat{n}} \hat{\sigma}_{A B}= & \hat{\sigma}_{A}{ }^{C} \hat{\sigma}_{C B}+\frac{1}{n-2} \hat{h}_{A B} \hat{\sigma}_{C D} \hat{\sigma}^{C D} \\
& -\frac{1}{\hat{\rho}}\left(\hat{\mathcal{D}}_{A} \hat{\mathcal{D}}_{B}-\frac{1}{n-2} \hat{h}_{A B} \hat{\mathcal{D}}^{2}\right) \hat{\rho},
\end{aligned}
$$

where $\hat{n}$ denotes the unit normal to the level set of $\tau$. Using $\nabla_{0}^{2} \tau=0$, we obtain

$$
\begin{aligned}
£_{\hat{n}} \hat{\mathcal{D}}_{A} \ln \hat{\rho} & =\hat{k} \hat{\mathcal{D}}_{A} \ln \hat{\rho}+\hat{\mathcal{D}}_{A} \hat{k}, \\
£_{\hat{n}} \hat{k} & =-\|\hat{\sigma}\|^{2}-\frac{1}{n-2} k^{2}-\frac{1}{\hat{\rho}} \hat{\mathcal{D}}^{2} \hat{\rho}, \\
£_{\hat{n}} \mathcal{D}_{A} \hat{k} & =\hat{\mathcal{D}}_{A} £_{\hat{n}} \hat{k}+\left(\hat{\mathcal{D}}_{A} \ln \hat{\rho}\right)\left(£_{\hat{n}} \hat{k}\right) .
\end{aligned}
$$

From the above equations, it can be seen that

$$
\hat{\sigma}_{A B}=0, \quad \hat{\mathcal{D}}_{A} \hat{\rho}=0, \quad \hat{\mathcal{D}}_{A} \hat{k}=0,
$$

that is, each level surface of $v$ is totally umbilic and hence spherically symmetric, which implies that the metric is isometric to those found in 17].

This is of course local result since we consider only the region containing no saddle points of the harmonic function $\tau$. To obtain the global result, we need a further assumption such as analyticity. However, the assumption that there is no saddle point may be justified as follows. At a saddle point $\rho=0$, the level surface of $\tau$ is multi-sheeted, that is the embedding of the level surfaces is singular there. One can find at least one level surface such that $k_{A B} \neq 0$ near the saddle point. Then, Eq. (25) implies that the saddle point is singular. If the horizon is not connected, this naked singularity must exist to compensate for the gravitational attraction between black holes.

One motivation for the present work was to embark on a programme of proving the uniqueness of static $p$-brane solutions. In general these take the form

$$
d s^{2}=e^{2 \gamma \Phi}\left(d \mathbf{y}_{p}^{2}\right)+e^{2 \delta \Phi} g_{\mu \nu} d x^{\mu} d x^{\nu} .
$$

Dimensional reduction on $\mathbf{E}^{p}$ will take the EinsteinHilbert action to the Einstein-Hilbert action if $(n-2) \delta+$ $p \gamma=0$. If the higher dimensional metric is coupled to an $n-2$ form $F_{n-2}$ with no scalars, we obtain the Lagrangian 18 


$$
R-2(\partial \phi)^{2}-\frac{2}{(n-2) !} e^{-\alpha \phi} F_{n-2}^{2},
$$

with

$$
\gamma^{2}=\frac{2(n-2)}{p(n+p-2)}
$$

and

$$
\alpha^{2}=\frac{8 p(n-3)^{2}}{(n-2)(n+p-2)} .
$$

We can now apply the results of this paper for $(n, p)=$ $(6,2)$ or $(5,3)$. For magnetic branes we need to make a duality transformation. We plan to give more details in a future publication.

We have shown that if space-time is assumed to asymptotically flat then the only regular static ellectric dilaton black holes are hyper-spherically symmetric and hence given the solutions in [17. However other, nonasymptotically flat, solutions may be obtained by replacing the metric of the round $(n-2)$-sphere by any other Einstein manifold whose Ricci-curvature has the same magnitude as that of a unit round $(n-2)$-sphere. In particular we can replace the round metric on $S^{5}, S^{6}, \ldots, S^{9}$ by the infinite sequences of Einstein metrics found recently by Bohm 12]. These have the form

$$
d \theta^{2}+a^{2}(\theta) g_{p}+b^{2}(\theta) g_{q}
$$

where $g_{m}$ is the unit round metric on the $m$-sphere, $p+q=n-3$ and neither $p$ nor $q$ is one. In general these metrics are inhomogeneous with isometry groups $S O(p+1) \times S O(q+1)$. The round metric on $S^{n-2}$ is given by $a=\sin \theta$ and $b=\cos \theta$. In addition, Bohm demonstrates the existence of infinite sequences of smooth Einstein metrics which converge to singular metrics of finite volume. By a theorem of Bishop [13], the volume of these metrics is always less than that of the round metric. In the vacuum case,it follows that for fixed temperature, the associated static black holes always have smaller Bekenstein-Hawking entropy than the spherical black hole. For this reason we believe that these metrics are all unstable. Preliminary results of GWG obtained with Sean Hartnoll show that the stability depends upon the spectrum of the Lichnerowicz operator for the Bohm metrics. This is not yet known. Presumably, if they are unstable, then they will decay to the spherical black-hole solution. An interesting question, which is beyond the scope of the methods of this paper because of the absence of a suitable positive energy theorem, is whether the Bohm black holes are unique among metrics with the same asymptotics, i.e. those constructed from the same Bohm metric.

One might question what physical relevance the Bohm metrics, not being asymptotically flat, might have. One possible application is that they may be used in D3branes solutions and applied to the AdS/CFT correspondence. The volume may then be related to the central charge of a conformal field theory [14]. In this way we obtain a further connection between the entropy of horizons, geometry and the central charges of quantum field theories where now Bishop's theorem provides a universal bound for the central charge.

Another possibility is that in modeling scattering and in connection with $\mathrm{TeV}$ black hole physics in the context of small extra dimensions, the appropriate boundary condition may be more general than the standard one of asymptotic flatness.

Acknowledgments. We would like to thank H. Kodama and H. S. Reall for useful discussion and comments. TS's work is partially supported by Yamada Science Foundation. GWG would like to thank M. Anderson for bringing [15] to his attention.

[1] R.C. Myers and M.J. Perry, Ann. Phys. 172, 304 (1986).

[2] P.C. Argyres, S. Dimopoulos and J. March-Russell, Phys. Lett. B441, 96 (1998); R. Emparan, G.T. Horowitz and R.C. Myers, Phys. Rev. Lett. 85, 499 (2000); S. Dimupoulos and G. Landsberg, Phys. Rev. Lett. 87, 161602 (2001); S.B. Giddings and S. Thomas, hepth/0106219

[3] W. Israel, Phys. Rev. 164, 1776 (1967).

[4] G.L. Bunting and A.K.M. Masood-ul-Alam, Gen. Rel. Grav. 19, 147 (1987).

[5] B. Carter, Phys. Rev. Lett. 26, 331 (1971); S.W. Hawking, Commun. Math. Phys. 25, 152 (1972); D.C. Robinson, Phys. Rev. Lett. 34, 905 (1975).

[6] B. Carter, and Black Hole Configurations." in Gravitation in Astrophysics, edited by B. Carter and J.B. Hartle (Plenum, New York, 1987); M. Heusler, Black Hole Uniqueness Theorems, (Cambridge Univ. Press, London, 1996).

[7] R. Emparan and H.S. Reall, Phys. Rev. Lett. 88, 101101 (2002).

[8] F.R. Tangherlini, Nuovo Cimento 27, 636 (1963).

[9] S. Kobayashi and K. Nomizu, Foundations of Differential Geometry, Vol. II (Interscience Publishers, New York, 1969), Sec. VII, Theorem 5.1.

[10] ibid. Theorem 6.4.

[11] E. Witten, Commun. Math. Phys. 80, 381 (1981).

[12] C. Bohm, Invent. Math 134, 145-176 (1998).

[13] R. L. Bishop, Notices Amer Math Soc 10363 (1963).

[14] S. Gubser, Phys. Rev. D 59, 025006 (1999).

[15] S. Hwang, Geometriae Dedicata 71, 5 (1998).

[16] A.K.M. Masood-ul-Alam, Class. Quantum Grav. 10, 2649 (1993).

[17] G.W. Gibbons, Nucl Phys B207 337 (1982),G. W. Gibbons and K. Maeda, Nucl Phys B298 741 (1988)

[18] G. W. Gibbons, G. Horowitz and P. K. Townsend, Class Quant Grav 12297 (1995) 